\title{
30 Jahre Mauerfall in der französischen Presse
}

\section{Stefanie Braun}

Université de Montpellier

\section{Abstract}

Der Beitrag analysiert das breite Echo, dass das Mauerfall-Jubiläum in der französischen Presse fand. Es handelt sich um eine Untersuchung von über hundertArtikeln, die in Frankreich um den 9. November in den, lautAuflistung der ACPM, bedeutendsten Tages- und Wochenzeitungen erschienen. Die herausgearbeiteten Thematiken behandeln die Darstellung und Interpretation des historischen Ereignisses in Frankreich, die Frage nach der Erinnerung und Vergangenheitsbewältigung der DDR, die veränderte Erinnerungsperspektive anlässlich des 30 . Jahrestags, die Bedeutung des Mauerfalls für die heutige Disparität zwischen dem Osten und dem Westen Deutschlands, den Zusammenhang zwischen 1989 und der europäischen Entwicklung und die Debatte über das Ende der Geschichte und der Ideologien. Die Untersuchung der französischen Presse zeigt die fortdauernde Bedeutung des Mauerfalls in den Debatten über Deutschland und Europa, wobei sich der Blick nunmehr auf das Ereignis außerhalb der deutschen Grenzen richtet, so dass sich das jetzige Mauerfall-Gedenken von der vorherigen Euphorie unterscheidet.

La contribution revient sur le retentissement de l'anniversaire de la chute du Mur de Berlin dans la presse française à travers l'étude de plus de cent articles parus autour du 9 novembre 2019 dans les principaux quotidiens et hebdomadaires français selon le classement de l'ACPM. Les thématiques dégagées se consacrent à la représentation et l'interprétation de l'événement historique en France, la problématique du traitement du passé et de la mémoire de la RDA, le changement de la perspective mémorielle, la relation entre la chute du Mur et la question européenne, la signification de la chute du Mur en lien avec les disparités est-ouest en Allemagne et les débats sur la fin de l'Histoire et des idéologies. Au-delà du simple constat d'une commémoration qui se démarquerait des précédentes par une célébration plus discrète, l'étude de la presse française démontre dans quelle mesure le regard porté sur l'évènement hors-frontières allemandes a évolué et à quel point il reste encore un enjeu idéologique et polémique dans les débats sur l'Allemagne et l'Europe dans la France actuelle.

This paper examines the wide echo about the anniversary of the Fall of the Berlin Wall in the French press through the analysis of more than hundred articles published around November 9th 2019 in the most relevant daily and weekly Papers according to the ranking of the ACPM. The contribution deals with several topics: the representation and interpretation of the historical event in France, the dealing with the GDR memory and the processes to overcome the past, the connection between the Fall of the Berlin Wall and Europe issues, the meaning of the event in relation with the east-west disparity in Germany and the ideological discussions about the end of History. More than the simple observation of an anniversary which stands apart from the previous ones, the study of the French press shows how polemical and ideological the Fall of the Berlin Wall remains in the French debates over Germany and Europe.

\section{Keywords}

Französische Presse $•$ Erinnerung $•$ Europa $・$ Mauerfall $•$ Gedenken

Presse française $\cdot$ mémoire $•$ Europe $\bullet$ chute du Mur • commémoration

French press $\cdot$ memory $\bullet$ Europe $\cdot$ Fall of the Wall $\bullet$ commemoration

Das Jubiläum des Mauerfalls 1989 hat in der französischen Presse ein breites Echo gefunden. Für den vorliegenden Beitrag wurden über hundert Artikel, die in Frankreich am 9. November 2019 und in der Woche vor dem Jahrestag des Mauerfalls erschienen sind, untersucht und rezensiert. Die Artikel waren in bedeutenden Tageszeitungen und wöchentlich erscheinenden Nachrichtenmagazinen veröffentlicht worden. Was ihre Verbreitung betrifft, so gehören die hier ausgewählten
Tageszeitungen, laut Auflistung der ACPM $^{1}$ für das Jahr 2019, zu den acht größten Vertretern der Tagespresse. Es handelt sich dabei um folgende Zeitungen: Le Figaro, Le Monde, Les Echos, La Croix, Libération, L'Humanité. Zur besseren Repräsentativität der französischen Printmedien

1 Die ACPM (Alliance pour les Chiffres de la Presse et des Médias) erhebt Statistiken über Verbreitung und Verteilung der Zeitschriften. 
wurden zusätzlich die Tageszeitung L'Opinion, wegen ihrer pro-europäischen Einstellung, und La Dépêche du Midi, als Beispiel einer regionalen Tageszeitung, ausgewählt. Bei den Wochenzeitschriften fiel die Wahl auf drei national vertriebene Nachrichtenmagazine, L'Express, L'Observateur, Le Point, sowie das monatlich erscheinende Blatt Le Monde diplomatique, das international weit verbreitet ist.

Durch diese breitangelegte Auswahl ist ein großes Spektrum an ideologischen Grundeinstellungen, die sich in der jeweiligen redaktionellen „Philosophie“ spiegeln, angesprochen, die von rechts bis linksextrem, von liberal bis äußerst kapitalismuskritisch reichen. Ziel der Untersuchung ist es, die Sichtweisen und Interpretationen in den Darstellungen, die dem Mauerfall als politisches und historisches Ereignis gewidmet sind, herauszuarbeiten. Zunächst sollen jedoch die ausgewählten Presseorgane kurz vorgestellt werden, um danach deren thematische Lektüre und Interpretation zu präsentieren.

Die von der ACPM durchgeführte „Rangfolge der Verbreitung nationaler Tagespresse in Frankreich für 2019“ basiert auf der Zählung der täglich von der jeweiligen Ausgabe verkauften Exemplare. An erster Stelle steht Le Figaro mit 325.938 Exemplaren. Das 1826 gegründete Blatt ist die älteste der heute noch veröffentlichten französischen Tageszeitungen. Die angestrebte redaktionelle Grundeinstellung ist liberal bis konservativ, europafreundlich, aber der nationalen Kultur verpflichtet, ${ }^{2}$ seine Leserschaft fühlt sich zur Rechten oder zur rechten Mitte zugehörig. Le Monde folgt mit 325.565 Exemplaren dicht auf Platz zwei der Tabelle für 2019. ${ }^{3}$ Die Zeitung wurde 1944 gegründet und ihre Orientierung liegt Mitte-Links wie auch ihre Stammleser. ${ }^{4}$ Mit 130.059 Exemplaren hält Les Echos, 1908 gegründet, den vierten Rang. ${ }^{5}$ Dieses Informationsblatt für Wirtschaft und Finanzen ist traditionell liberal und pro-europäisch, Verfechter der Marktwirtschaft und dem internationalen Geschehen zugewandt. ${ }^{6}$ Die 1883 gegründete Tageszeitung La Croix ist katholisch und bekennt sich, trotz Änderungen in jüngster

2 „Libéral mais pas dogmatique, conservateur mais pas passéiste, européen mais pas eurobéat, attaché à défendre la culture française mais ouvert sur le monde“" (Liberal, doch nich dogmatisch, konservativ aber nicht rückwärtsgewandt, europäisch aber nicht von einem ein fältigen Europaoptimismus ergriffen, der französischen Kultur verpflichtet aber weltoffen) Brézet, Alexis, Leitartikel, 28.03.2013, https://www.lefigaro.fr/mon-figaro/2013/03/27/10001 20130327ARTFIG00729--le-figaro-se-reinvente.php.

3 Le Monde steht auch manchmal an erster Stelle. Vgl. Bonacossa, Caroline, ,Le Monde quotidien le plus vendu en France en avril 2019“, 12.06.2019, https://www.strategies.fr/actualites/medias/4030535W/le-monde-quotidien-le-plus-vendu-en-france-en-avril-2019.html.

451 \% der Linkswähler bezeichnen sich 2014 als Leser von Le Monde. „IFOP en partenariat avec Marianne. Sondage du 04/04/2014. Analyse du vote au premier tour des élections municipales en fonction des habitudes médias", https://www.ifop.com/publication/ analyse-du-vote-au-premier-tour-des-elections-municipales-en-fonction-des-habitudesmedias/.

5 Die Sporttageszeitung L'Equipe steht an dritter Stelle mit 233.791 verkauften Exemplaren.

6 „quotidien économique ouvert sur l'international et les grandes entreprises“, „libéral européen et résolument opposé à l'économie étatisée“ („eine weltoffene und unternehmerfreundliche Wirtschaftstageszeitung“, „liberal, europäisch und entschieden gegen staatlich Eingriffe in die Wirtschaft"), Gaston-Breton, Tristan, "Les Echos': un nouveau média qui n'a de cesse de se réinventer", 12.10 .2018 , https://www.lesechos.fr/idees-debats/editos-analyses/les-echos-un-media-qui-na-cesse-de-se-reinventer-141653, „favorable à l'économie de marché et à la liberté d'entreprendre" (für die Marktwirtschaft und die Unternehmerfreiheit), https://www.lesechos.fr/ethical-code.
Vergangenheit, zur christlichen Identität. ${ }^{7}$ Mit 87.682 Exemplaren ist sie die sechstgrößte nationale Tageszeitung in punkto verkaufter Auflage. Libération, ursprünglich mit Unterstützung von Jean-Paul Sartre 1974 gegründet, heute an die linke Mitte und die sozialdemokratische Linke gerichtet, steht mit 71.466 Exemplaren an siebter Stelle. Mit 36.621 verkauften Exemplaren auf französischem Staatsgebiet ist L'Humanité die achtgrößte nationale Tageszeitung. 1904 von Jean Jaurès ins Leben gerufen, ist sie auf der äußeren Linken angesiedelt und steht weiterhin der Kommunistischen Partei nahe, nachdem sie von 1920 bis 1994 deren zentrales Organ gewesen war. ${ }^{8}$ Bei 41.000 Exemplaren hat die 2013 gegründete Tageszeitung L'Opinion eine klare publizistische Linie und bezeichnet sich als „liberal, unternehmerfreundlich, europäisch". ${ }^{9}$ Schließlich ist La Dépêche du Midi mit 125.824 Exemplaren die elftgrößte regionale Tageszeitung.

Unter den französischen Wochenzeitschriften verkauft das 1972 gegründete und Mitte-Rechts stehende Nachrichtenmagazin Le Point 292.795 Exemplare pro Ausgabe. Dicht darauf folgt mit 215.877 Exemplaren L'Obs, 1964 unter dem Namen Le Nouvel Observateur ${ }^{10}$ gegründet. Das Blatt rechnet sich selbst zur sozialdemokratischen Bewegung. ${ }^{11}$ Die Wochenzeitschrift L'Express, die sich der europäischen Idee und der Öffnung der Märkte gegenüber positiv äußert, wurde 1953 ins Leben gerufen und hat eine Auflage von 201.126 Exemplaren. Die 1954 gegründete Monatszeitschrift Le Monde diplomatique verfügt 2019 über eine Verbreitung von 143.872 zahlenden Lesern. Sie wird in 19 Sprachen übersetzt, behandelt hauptsächlich internationale Themen und bekennt sich zu einer kritischen Einstellung gegenüber Kapitalismus, Freihandel und Militarismus. ${ }^{12}$

Legt man diesen ausgewählten Medien mit dem Schwerpunkt Mauerfall eine detaillierte und thematisch ausgerichtete Untersuchung zugrunde, so können sechs Schwerpunkte herausgearbeitet werden. Ein erster Schwerpunkt (die Hälfte der Artikel, wobei es oft Überschneidungen mit einem der anderen Schwerpunkte gibt) liegt auf punktuellen historischen Ereignissen, die im Zusammenhang mit dem Tagesablauf am 9. November oder in den Wochen vor dem Mauerfall stehen. Oft wird Zeitzeugen dieser Ereignisse das Wort gegeben, dabei auch Journalisten, die sich zum Zeitpunkt des Mauerfalls in Berlin aufgehalten hatten. Mitunter sind es auch historische Persönlichkeiten, denen, wie Michail Gorbatschow,

\footnotetext{
7 „un quotidien qui affirme clairement son identité catholique mais refuse de s'y enfermer", (eine Tageszeitung, die offen zu ihrer katholischen Identität steht, ohne sich auf sie zu beschränken), Carasco, Aude, "La Croix marque sa différence avec une nouvelle formule“, 19.01.2016, https://www.la-croix.com/Economie/Medias/La-Croix-marque-difference-avecnouvelle-formule-2016-01-19-1200732198.

8 Vgl. Clérin, Cédric, „Les 1001 naissances de l'Humanité“, 18.04.2019, https://www.humanite.fr/presse-les-1-001-naissances-de-Ihumanite-671054.

9 „libérale, pro-business, européenne“, https://www.lopinion.fr/qui-sommes-nous.

10 Die Zeitung trägt bis 2014 diesen Namen.

11 „La charte“, 15.12.2006, https://www.nouvelobs.com/medias/20061215.OBS3094/lacharte.html.
}

12 https://www.monde-diplomatique.fr/diplo/apropos/. 
eine herausragende Rolle in jener Zeit zukam. Ein zweites wichtiges Thema (25\% der Artikel) ist die Erinnerung und die Frage nach der „Vergangenheitsbewältigung“ der DDR. Die heutige Disparität zwischen dem Osten und dem Westen Deutschlands ist eine dritte, oft behandelte Problematik $(20 \%$ der Artikel). In einer vierten Gruppe von Themen wird ebenfalls das Gedenken an den Mauerfall debattiert, jedoch mit Blick auf die veränderte Erinnerungsperspektive anlässlich des 30. Jahrestages (15\% der Artikel). Europa, seine Zukunft, seine Schwerpunkte und deren Zusammenhang mit dem Mauerfall bilden den fünften behandelten Themenkreis (25\%). Der sechste Punkt betrifft die ideologische Debatte über das „Ende der Geschichte“ und die Auslegung des Mauerfalls als richtungsweisendes Ereignis im Zusammenhang mit der Entwicklung und der Entstehung des Modells der pluralistischen Demokratie (15\% der Artikel).

Unter den Artikeln, deren Schwerpunkt auf dem geschichtlichen Überblick der Ereignisse liegt, behandelt eine kleine Zahl die Geschichte der Berliner Mauer, ihre Errichtung $^{13}$ oder den Handlungsablauf am 9. und 10. November 1989. ${ }^{14}$ Die Mehrheit der Artikel erwähnt eher die ersten Risse im Eisernen Vorhang und die Rolle der ehemaligen Satellitenstaaten des Ostblocks von März bis November 1989, die Durchtrennung des Eisernen Vorhangs an Ungarns Grenze, das paneuropäische Picknick in Sopron ${ }^{15}$ oder die Besetzung der bundesdeutschen Botschaft in Prag $^{16}$. Die Tendenz, Themen und bekannte Persönlichkeiten des ehemaligen Ostblocks in den Vordergrund zu stellen, wird auch in den zahlreichen Gesprächen mit Leitfiguren und Politikern der kommunistischen Länder deutlich, wie in Interviews und Porträts nicht nur von Michail Gorbatschow ${ }^{17}$ und seinen engen Beratern ${ }^{18}$, sondern auch von Lech

13 Vaulerin, Arnaud, "La nuit où les Soviétiques ont fait le Mur", in Libération, Dossier Berlin 30 ans, l'Âge Mur" (27-36), Nr. 11948, 2-3.11.2019, 34-35.

14 Rousseaux, Nicolas, „Le 9 novembre 1989. Une journée très particulière“, in Les Echos Week-end, Supplément au n ${ }^{\circ} 23071$, Les Echos, 8.-9.11.2019, 36-37; Avril, Pierre, .,9 novembre 1989: le jour qui a transformé l'Europe", in Le Figaro, Nr. 23401,9-10.11.2019, 2-3; Piérot, Jean-Pierre, ,Le 9 novembre 1989, les retrouvailles allemandes“", in L'Humanité, $\mathrm{Nr}$. 22803, 8.-10.11.2019, 6-7; Dorman, Veronika, Et pendant ce temps, à Moscou, Gorbatchev dormait", in Libération, Dossier „Berlin 30 ans, l'Âge Mur“, Nr. 11951, 6.11.2019, 6-7.

15 Haquet, Charles, „Hongrie. Le jour où le Rideau de fer s'est enfin déchiré“, in L'Express, Dossier „Mur de Berlin. L'Europe, 30 ans après“ (26-55), Nr. 3566, 6-12.11.2019, 40-44; Didelot, Nelly, „Le Rideau de fer déchiré à la frontière hongroise“, Libération, Dossier „Berlin 30 ans, l'Âge Mur", Nr. 11950, 5.11.2019, 14-15; Lorrain, François-Guillaume, „Le piquenique“, in Le Point, Dossier "Chute du Mur, Histoire inattendue“ (52-61), Nr. 2463, 7.11.2019, $56-59$

16 Massiot, Aude, „A Prague, l'ambassade de RFA ouvre une brèche“, in Libération, Dossier "Berlin 30 ans, l'Âge Mur", Nr. 11952, 7.11.2019, 8-9.

17 Gorbatchev, Mikhaïl, „Nous avons pris la seule décision acceptable“, (das Gespräch führte Laure Mandeville), Le Figaro, (wie Anmerkung 14), 8; Mandraud, Isabelle, Le testament ambivalent de Mikhaïl Gorbatchev“, in Le Monde, Nr. 23273, 7.11.2019, 29.

18 Fedorovski, Vladimir, ,Le soir de la chute du Mur, Gorbatchev aurait demandé qu'on ne le réveille pas", (das Gespräch führte Gilles Sengès), in L'Opinion. Quotidien libéral//politique, économie, international, Nr. 1629, 8-9.11.2019, 8; Fédorovski, Vladimir, „Les derniers secrets du mur", in L'Express, (wie Anmerkung 15), 48-49, Dossier "Mur de Berlin. L'Europe, 30 ans après; Gratchev, Andreï, „En 1989, personne n'était préparé à ce bouleversement historique", in Le Monde, Nr. 23273, (wie Anmerkung 17), 28.
Walesa ${ }^{19}$ oder anderen polnischen Politikern. ${ }^{20}$ Diese Art, die Aufmerksamkeit auf das Verdienst Polens, Ungarns und der Tschechoslowakei als Vorreiter der 1989er-,Revolution“ zu lenken, ist eine Parallele zur Haltung der deutschen Regierung. Schließlich waren bei den Berliner Feierlichkeiten zum 30. Jahrestag des Mauerfalls nur die Präsidenten Polens, Ungarns, der Slowakei und der Tschechischen Republik zugegen. Die Abwesenheit westlicher Regierungsvertreter kommentierte vor allem L'Express. ${ }^{21}$ Neben der Befragung großer politischer Persönlichkeiten werden auch Zeugen aus künstlerischen und intellektuellen Milieus der DDR in den Vordergrund gestellt, am weitaus häufigsten Dissidenten und Regimekritiker, so etwa der Schriftsteller Christoph Hein in der Zeitung La Croix, der dort als „klarsehender Chronist"22 (chroniqueur lucide) eines von der Ost-WestFraktur gekennzeichneten Landes beschrieben ist, oder auch Wolf Biermann. ${ }^{23}$ Manche der Befragten sind aus der Zeit nach dem Mauerfall bekannt. Es überwiegen Überlegungen der Verfasser zu Unwägbarkeiten und Fehlern im Wiedervereinigungsprozess. Ein Beispiel dafür ist das Interview mit dem ehemaligen Pressesprecher der Treuhandgesellschaft Wolf Schöde. ${ }^{24}$

Der Verlauf des Mauerfalls wird von anderen Journalisten so wiedergegeben, wie sie inn 1989 erlebt hatten. ${ }^{25}$ So überlässt Libération den Reportern und Fotografen das Wort, die im November 1989 zugegen waren. ${ }^{26}$ Die diachrone Perspektive ermöglicht dementsprechend eine vergleichende Sicht auf die Zeit vor und nach der Wende mit dem Vorschlag einer Neuauslegung des Mauerfalls, wobei die Gefahr besteht, dass dabei eine teleologische Sichtweise der Vergangenheit entsteht. L'Obs traf die Wahl, Artikel wieder zu veröffentlichen, die schon in älteren Ausgaben

19 Walesa, Lech, "Les Polonais ont cassé quelques dents à l'ours soviétique" (das Gespräch führte Justine Salvestoni), in Libération, Dossier „Berlin 30 ans, l'Âge Mur“, Nr. 11949, 4.11.2019, 6-7; Walesa, Lech „Je pensais être le dernier révolutionnaire de mon pays“" (das Gespräch führte Jean-Baptiste François, Übersetzung Magdalena Viatteau pays", (das Gesprach führte Jean-Baptiste François, Ubersetzung Magdalena Viatteau, „Portrait. Un homme controversé mais populaire“, ebd., 17; François, Jean-Baptiste, „Lech "Portrait. Un homme controverse.

20 Kwasniewski, Aleksander, „L'année 1989 est la meilleure de l'histoire de la Pologne“, (das Gespräch führte Clément Daniez), in L'Express, Dossier „Mur de Berlin. L'Europe, 30 ans après", (wie Anmerkung 15), 50-51; Michnik, Adam, „C'est en Pologne, avec Solidarnosc, que le mur de Berlin s'est fissuré", in Le Monde, Nr. 23274, 8.11.2019, 24

21 „L'Allemagne commémore la chute du mur de Berlin“, 9.11.2019, https://www.lexpress. fr/actualite/monde/europe/comment-l-allemagne-commemore-la-chute-du-mur-du-berlin_2106346.html.

22 Alençon, François, „D'une Allemagne à l'autre“, in La Croix, Nr. 41552, 7.11.2019, 26-

23 Biermann, Wolf, „Wolf Biermann, entretien 'Mme Liberté n'embrasse pas M. Barbelés' (das Gespräch führte Thomas Wieder), in Le Monde, Nr. 23274, (wie Anmerkung 20), 25.

24 Schöde, Wolf, "La plus grande erreur a certainement été le facteur humain“, (das Gespräch führte Ninon Renaud), in Les Echos, Nr. 23071, (wie Anmerkung 14), 7.

25 Chalier, Pierre, „Il y a 30 ans, la chute du Mur de Berlin“, in La Dépêche du Midi, 9.11.2019, 2-3; André, Luc, „Est-Ouest: Comment réduire la facture allemande?", in L'Opinion, (wie Anmerkung 18), 1-3, hier 2; Joffrin, Laurent, ,"Trouble nostalgie“, Leitartikel, in Libération, Nr. 11954, 9.-11.2019, 2.

26 Auffray, Alain, „Cette nuit-là, je n'ai pas eu besoin de mon passeport", in Libération Dossier „Berlin 30 ans, l'Âge Mur", Nr. 11953, 8.11.2019, 6-7; Weiss, Maurice, „'Berlin-Est était comme un autre pays. II y avait du bordel partout'" (das Gespräch führte Johanna Luyssen), (wie Anmerkung 13), 32-33. 
zwischen 1989 und 1994 abgedruckt worden waren, ${ }^{27}$ mit der Absicht, geschichtliche Dokumente (documents d'histoire immédiate), ${ }^{28}$ Artikel, Reportagen und Gespräche mit herausragenden Persönlichkeiten ${ }^{29}$ auszugraben. Durch den Vergleich zwischen den älteren Artikeln und denen, die im November 2019 erschienen sind, lässt sich ebenfalls feststellen, dass den zwei Wochen vor dem Mauerfall eine große Bedeutung beigemessen wurde, um die Idee zu betonen, dass der Ablauf der Ereignisse unausweichlich war. ${ }^{30}$ Zudem ergibt sich manchmal aus dieser Gegenüberstellung und parallelen Betrachtung die Diskrepanz zwischen der damaligen Geisteshaltung und der Bedeutung, die im Nachhinein dem Mauerfall zugeschrieben wurde, wodurch Deutungsverschiebungen imzeitlichen Verlaufhervorgehoben werden. So schildert ein Artikel vom 2.-3. November 2019, wie die Schriftstellerin ${ }^{31}$ und Korrespondentin von Libération, Pascale Hughes, nach der historischen Pressekonferenz von Günter Schabowski versucht hatte, ihre Pariser Redaktion zu erreichen; die Redaktion schien jedoch kaum am Besuchsvisum für Ostbürger interessiert gewesen zu sein, denn die Korrespondentin erhielt als Antwort: „Nun beruhige dich, wir streiken gerade“ („Calme-toi on est en grève"). ${ }^{32}$ Am nächsten Tag erschien keine Ausgabe von Libération, aber die Zeitung machte es am Samstag den 11. November 1989 durch die Veröffentlichung einer 17 Seiten langen Doppelausgabe wieder wett. Im Gegensatz dazu geben andere Zeitungen an, einen historischen Moment und das Gefühl vom Ende einer Epoche oder gar eines Jahrhunderts sehr bewusst erlebt zu haben. ${ }^{33}$ Die Tendenz zur Historisierung der ideologischen Debatten, da sie mit den Begriffen vom Ende des Jahrhunderts und der Ideologien in Verbindung gebracht wird, ist übrigens ein Leitmotiv in der französischen Presse wie noch zu zeigen sein wird.

Die dritte Gruppe von Artikeln behandelt die Vergangenheit der DDR und ihre spezifische Identität. Diese Artikel thematisieren einerseits das Überwachungssystem in der DDR ${ }^{34}$, andererseits die Problematik der ostdeutschen Erinnerung und kollektiven Identität, vor allem in den zahlreichen Artikeln

27 Muller, Marie / Stern, Jean, „Jours tranquilles à Berlin-Est. Paru dans le $n^{\circ} 838$ 01/12/1980", in "Cahier Historique. Mur de Berlin. Le jour où le monde a basculé" (1-48) in L'Obs, Cahier $n^{\circ} 1$ de l'édition, $n^{\circ} 2870,7-13.11 .2019$, 8-11; Caviglioli, François, „Nuits blanches à Varsovie. Paru dans le n¹028, 20/7/1984", ebd., 12-17; Mari, Jean-Paul, "Le printemps de Leipzig“. Paru dans le n¹303, 26/10/1989“, ebd., 20-21; Muller, Marie, "Le grand bal des cousins pauvres. Paru dans le n ${ }^{\circ} 1306,16 / 11 / 1989^{\prime \prime}$, ebd., 22-25; Schlosser, François, „Les vieux ennemis de la liberté. Paru dans le n¹317, 1/2/1990“, ebd., 40-43.

28 „Voici, ce que nous écrivions sur le moment. Des documents d'histoire immédiate, qu disaient l'espoir immense - et, déjà, la vigilance“, Jauvert, Vincent, "Le Mur et nous“ (1-48), in L'Obs, Dossier "Cahier Historique. Mur de Berlin. Le jour où le monde a basculé“, (wie Anmerkung 27), 3 .

29 Havel, Václav, „Václav Havel. Le théâtre de la Dissidence. Paru dans le ${ }^{\circ} 1288$ 13/07/1989" (das Gespräch führte Elisabeth Schemla), ebd., 18-19.

30 Jauvert, Vincent, "Les seize jours qui ont changé le monde. Paru dans le ${ }^{\circ} 1565$ 3/11/1994", ebd., 30-35.

31 Vgl. u.a. La Robe de Hannah. Berlin 1904-2014, Paris, Les Arènes, 2014

32 Péron, Didier, „Calme-toi, on est en grève“, in Libération, Dossier „Berlin 30 ans, l'Âge Mur", (wie Anmerkung 13), 36.

33 Daniel, Jean, „L'Adieu au siècle. Paru dans le n¹317, 1/2/1990“, (wie Anmerkung 27), 36-39.

34 De Barochez, Luc , „Le puzzle secret de la Stasi“, in Le Point, Dossier „Chute du Mur, Histoire inattendue", (wie Anmerkung 15), 60-61. über Nicolas Offenstadts Buch Urbex RDA. ${ }^{35}$ Auch in Artikeln, die nicht ausschließlich diesem Autor gewidmet sind, wird er oft erwähnt. ${ }^{36}$ Darüber hinaus wird das Thema der ostdeutschen Identität auch in Abhandlungen behandelt, die die Frage aufwerfen, wie sich der Alltag und die Vergangenheit der ehemaligen DDR-Bürger am zutreffendsten ausdrücken ließe. Dies wird z.B. in Libération ${ }^{37}$ in einem Interview mit Hélène Camarade zu ihrem Buch Les Mots de la RDA ${ }^{38}$ über Humor und Witze in der DDR deutlich.

Die zweite Gruppe von Artikeln macht den Wiedervereinigungsprozess und die anhaltende Ost-West-Disparität zum Gegenstand der Untersuchung, wobei die Gewinne der AfD in den Gebieten der ehemaligen DDR ausdrücklich unterstrichen werden. ${ }^{39}$ Die Aufmerksamkeit wird auf die Spaltung und das Unbehagen eines „zusammengeflickten Landes" gelenkt, in dem sich die ehemaligen Bürger Ostdeutschlands als zweitklassige Bürger vorkämen. ${ }^{40} \mathrm{Oft}$ kommt diese Feststellung in Reportagen zum Ausdruck. ${ }^{41}$ Noch weiter gehen andere Artikel, die die Art und Weise offen kritisieren, wie die Wiedervereinigung verlief ${ }^{42}$ und dabei vor allem die Rolle der Treuhand anprangern. ${ }^{43}$ Manche Artikel vertreten die These einer Annektierung der DDR durch die Bundesrepublik, wobei die ostdeutsche Wirtschaft und Gesellschaft als Opfer einer Liquidierungsstrategie dargestellt werden. Diese oft formulierte These ${ }^{44}$ beruht auf einer

35 Offenstadt, Nicolas, Urbex RDA. L'Allemagne de l'Est racontée par ses lieux abandonnés, Paris, Albin Michel, 2019; Offenstadt, Nicolas, „Ex-RDA, l'histoire par les friches. Internés, Paris, Albin Michel, 2019; Offenstadt, Nicolas, „Ex-RDA, l'histoire par les friches. Inter-
view de Nicolas Offenstadt“, (das Gespräch führte Catherine Calvet), in Libération, Dossier view de Nicolas Offenstadt“, (das Gespräch führte Catherine Calvet),

36 Wieder, Thomas, „En Allemagne, la double-vie de l'ex-RDA“, in Cahier du Monde „30 ans après la chute du Mur", Nr. 23276, 10.-13.11.2019, 2-3, hier 3.

37 Camarade, Hélène, „En RDA, l'humour permettait une distance critique, jouer avec le langage soulignait l'écart entre le discours officiel et réalité", (das Gespräch führte Emmanuèle Peyret), in Libération, Nr. 11954, (wie Anmerkung 25) 22-23.

38 Camarade, Hélène / Goepper, Sybille, Les Mots de la RDA, Toulouse, Presses universitaires du Midi, 2019

39 Chalier, Pierre, „ll y a 30 ans, la chute du Mur de Berlin“, in La Dépêche du Midi, (wie Anmerkung 25), 2-3, hier 3; Rioux, Philippe, ,L'Allemagne de l'Est de la dictature à la démocratie, de la liberté aux disparités", ebd., 2; Fekl, Matthias, "Le 9 novembre, journée européenne de la liberté“, in L'Opinion, (wie Anmerkung 18), 4 ; Odent, Bruno, ,La Thuringe malade d'une réunification qui n'a pas eu lieu“, in L'Humanité, (wie Anmerkung 14), 5-6, hie 5; Pièt, Jean-Pierre, "Le 9 novembre 1989, les retrouvailles allemandes', in L'Humanité, (wie Anmerkung 14), Nr. 22803, 6-7, hier 7; Rioux Philippe, „'Allemagne de l'Est, de la dictature à la democratie, de la liberté aux disparités", in La Dépêche du Midi, (wie Anmerkung 25), 2; André, Luc, „Est-Ouest: Comment réduire la facture allemande?", in L'Opinion, (wie Anmerkung 18), 1-3, hier 2; Wieder, Thomas, „En Allemagne, la double-vie de l'ex-RDA“, in Cahier du Monde „30 ans après la chute du Mur", Nr. 23276, 10.-12.11.2019, 2-3; Grésillon, Boris, „Un mur peut en cacher un autre. En ex-RDA, des citoyens de seconde zone“, in $L e$ Monde diplomatique, Nr. 788, November 2019, 16-17.

40 Saint-Paul, Patrick, En Allemagne de l'Est, l'étrange mal de vivre des Ossis", in Le Figaro, 9.-10.11.2019, Nr. 23401, 4-5; Saint-Paul, Patrick, „Platzeck: 'La séparation était Figaro, 9.-10.11.2019, Nr. 23401, 4-5; Saint-Paul, Patrick, „Platzeck: 'La s'
plus profonde que nous ne le pensions', Le Figaro, (wie Anmerkung 14), 5.

41 Odent, Bruno, „La Thuringe malade d'une réunification qui n'a pas eu lieu“, in L'Humanité, (wie Anmerkung 14), 5-6; Hauteville, Jean-Michel, „Le pari gagné d'léna, 'ville lumière' de Thuringe", in Le Monde, Nr. 23272, 6.11.2019, 15; Hauteville, Jean-Michel, „A l'est de l'Allemagne la réunification n'a pas été si florissante", ebd., 14-15; Hauteville, JeanMichel, „Le retour au pays des 'revenants'“, ebd., 14.

42 Collomp, Florentin, „Le titanesque défi inachevé de la réunification économique“, in Le Figaro, (wie Anmerkung 14), 6-7; Odent, Bruno, ,La Thuringe malade d'une réunification qui n'a pas eu lieu“', in L'Humanité, (wie Anmerkung 14), 5-6.

43 Philippot, David, "Quand les 'hommes en noir' de la Treuhand liquidaient les usines de I'Est", in Le Figaro, (wie Anmerkung 14), 6-7; Knaebel, Rachel / Rimbert, Pierre, „Liquider une société", in Le Monde diplomatique, (wie Anmerkung 39), 14

44 Knaebel, Rachel / Rimbert, Pierre, „Il y a trente ans, la chute du Mur de Berlin. Allemagne de l'Est, histoire d'une annexion", in Le Monde diplomatique, (wie Anmerkung 39), 1, 14-15; Chalier, Pierre, „Il y a 30 ans, la chute du Mur de Berlin“, in La Dépêche du Midi, (wie 14-15; Chalier, Pierre, 
Darlegung von Argumenten, die das Bild einer hinkenden und asymmetrischen Wiedervereinigung aufzeigen, von der vor allem Westdeutschland profitiert hätte. ${ }^{45}$

In ihrer Betonung der Diskrepanz zwischen Ost und West und der Notwendigkeit, das Erbe der Wiedervereinigung neu zu überdenken, schlägt sich der Versuch der französischen Presse nieder, sich als Echo der deutschen Presse zu präsentieren. Wenn die Journalisten eine Veränderung der Erinnerungsperspektive in Deutschland feststellen, beziehen sie sich insbesondere auf die Studien von Raj Kollmorgen. Allgemein wird festgestellt, dass das jetzige MauerfallGedenken sich durch das Eingeständnis der aktuellen Schwierigkeiten in einem gespaltenen Deutschland von der vorherigen Euphorie unterscheidet. ${ }^{46}$ Die Hoffnung, der deutschen Teilung den Rücken gekehrt zu haben und eine Welt erreicht zu haben, die den Triumph der liberalen Demokratie über die Diktaturen feiern würde, habe sich als illusorisch erwiesen, was die Zurückhaltung bei den Gedenkfeierlichkeiten erkläre. ${ }^{47}$ Wenn dieser Mangel an Begeisterung von manchen kritisiert wird, die ihrerseits den Triumph von Demokratie ${ }^{48}$ und Freiheit ${ }^{49}$ bejubeln wollen, denunzieren andere einen „Gedenkzombie ${ }^{50}$ (zombie mémoriel) und die Tatsache, dass vor allem die Besitzenden feierten. ${ }^{51}$ Die Kritik betrifft auch die Mauer als Memorial-Relikt: Mehrere Journalisten prangern die Kommerzialisierung der Geschichte der Mauer an, weil das ein tieferes Reflektieren über ihre Bedeutung verhindere. ${ }^{52}$

Die Auslegung des Mauerfalls als entscheidendes Ereignis für die Weiterentwicklung der Europäischen Union ist in den untersuchten Artikeln allgegenwärtig; Europa, seine Identität und seine Gegensätze bilden somit den Hintergrund der Überlegungen. Durch das Porträtieren von Zeitzeugen und der Schilderung der Folgen des Mauerfalls wird Europa entweder gefeiert oder in Frage gestellt. Manchmal wird der Zusammenhang nur angedeutet, wie etwa im Porträt des Cellisten Rostropowitsch, von dem es heißt, er habe bei seinem Konzert an der Mauer vor allem als Europäer

45 Knaebel, Rachel / Rimbert, Pierre, „Vieilles dettes et gros bénéfices“, in Le Monde diplomatique, (wie Anmerkung 39), 15.

46 Renaud, Ninon, „Berlin fête les trente ans de la chute du Mur sans euphorie“, Les Echos, (wie Anmerkung 14), 7; Knaebel Rachel / Pierre Rimbert, „Il y a trente ans, la chute du Mur de Berlin. Allemagne de l'Est, histoire d'une annexion“, in Le Monde diplomatique, (wie Anmerkung 39), 1, 14-15, hier 1.

47 Avril, Pierre, „Des commémorations empreintes de discrétion“, in Le Figaro, (wie Anmerkung 14), 3.

48 Fekl, Matthias, "Le 9 novembre, journée européenne de la liberté", in L'Opinion, (wie Anmerkung 18), 4; Jauvert, Vincent, „Le Mur et nous“, in L'Obs, Dossier "Cahier Historique. Mur de Berlin. Le jour où le monde a basculé“, (wie Anmerkung 27), 3.

49 Leitartikel, "Mur de Berlin: ne nous trompons pas de funérailles“, in Le Monde, $\mathrm{Nr}$ 23276, (wie Anmerkung 39), 26.

50 Knaebel, Rachel / Rimbert, Pierre, „Il y a trente ans...“, vgl. Anmerkung 44, 15

51 Ebd

52 Chalier, Pierre, „Il y a 30 ans, la chute du Mur de Berlin“, in La Dépêche du Midi, (wie Anmerkung 25), 2-3; Hauteville, Jean-Michel, „Berlin capitalise sur le tourisme du Mur", in Le Monde, Nr. 23275, 17; Gyldén, Axel / Bourdoiseau, Christophe, „A la recherche du Mur", in L'Express, Dossier "Mur de Berlin. L'Europe, 30 ans après“", (wie Anmerkung 15), 28-32 Gyldén, Axel / Bourdoiseau, Christophe, „Berlin, sans cesse réinventée“, ebd., 34-35; Gyldén, Axel, ,Le Mur à travers le monde“, ebd., 36-39. auftreten wollen. ${ }^{53}$ Der Fall der Berliner Mauer wird gewöhnlich als „Gründungsmythos der Europäischen Union“54 (Mythe fondateur de l'Union européenne), als „Sockel für Europa"55 (socle pour l'Europe) oder als Motor der deutschfranzösischen Freundschaft ${ }^{56}$ bezeichnet. Das Andenken an den Mauerfall erscheint dann als entscheidender politischer Beitrag zum Zusammenhalt und zur Zukunftsperspektive Europas. Demzufolge wird der 9. November in der proeuropäischen Tageszeitung L'Opinion als europäischer Tag der Freiheit charakterisiert, an dem man von nun an um jeden Preis feiern solle. ${ }^{57}$ In die gleiche Richtung weisen Le MondeArtikel, die an zwei für die aktuelle Geschichtsschreibung bedeutsamen Tagen erschienen: Der Leitartikel vom 10.12. November ruft die Leser auf, sich an die positiven Veränderungen, die der Mauerfall nach sich zog, zu erinnern, anstatt einer pessimistischen Europasicht nachzugehen ${ }^{58}$; einige Tage vorher, am 4. November 2019, zitiert Le Monde Heiko Maas, der die deutsch-französische Freundschaft als Motor für den Aufbau Europas beschwört. ${ }^{59}$ Manchmal ist der Ton pessimistischer, denn der Jahrestag des Mauerfalls bietet ebenfalls die Gelegenheit zur Diagnose von Spaltungen und Bruchstellen $^{60} \mathrm{im}$ gegenwärtigen Europa. ${ }^{61}$ Die Idee einer bruchstückhaften europäischen Identität in einem Kontinent, der dabei wäre, seine Wertmaßstäbe zu verlieren, steht hinter sehr vielen Artikeln und Reportagen über die Länder des ehemaligen sowjetischen Lagers. ${ }^{62}$ Dargelegt wird, wie populistische Regime die europäische Identität und die Erinnerung an den Mauerfall instrumentalisieren würden. Dabei wird auch eine Verbindung zwischen dem Zerfall des ehemaligen Ostblocks und dem Erstarken von Populismus, Rechtsextremismus und Identitätsbewegungen hergestellt. ${ }^{63}$ Enttäuschungen, Ängste und Misstrauen der Osteuropäer

53 Cojean, Annik, ,Le Maestro et le Mur", in Le Monde, Nr. 23276, (wie Anmerkung 39), 15. 54 Grésillon, Boris, vgl. Anmerkung 39.

55 Barnavi, Elie, „Le Mur, un socle pour l'Europe“, in L'Express, Dossier „Mur de Berlin. L’Europe, 30 ans après", (wie Anmerkung 15), 52-53.

56 Makarian, Christian, ebd., 27

57 Fekl, Matthias, „Le 9 novembre, journée européenne de la liberté“, in L'Opinion, (wie Anmerkung 18), 4 .

58 Leitartikel, „Mur de Berlin: ne nous trompons pas de funérailles“, in Le Monde, Nr. 23276, (wie Anmerkung 39), 26.

59 Maas, Heiko, „Où étiez-vous lorsque le mur de Berlin est tombé?", in Le Monde, $\mathrm{Nr}$. 23270, 4.11.2019, 28.

60 Salles, Alain, "Les fractures de l'Europe“" in Cahier du Monde „30 ans après la chute du Mur", Nr. 23276, (wie Anmerkung 39), 1.

61 Chastand, Jean-Baptiste / Mendraud, Isabelle / Ricard, Philippe, „Les chemins sinueux de la réunification européenne", ebd., 4.

62 Chastand, Jean-Baptiste, „Klara Ungar, symbole de la défaite des dissidents libéraux hongrois“, ebd., 5; Iwaniuk, Jakub, „En Pologne, la bataille mémorielle continue de faire rage“, ebd., 5; Mandraud, Isabelle, „En Lettonie, un entêtant parfum de guerre froide“, ebd., 6-7; Semo, Marc, ,Le procès d'Ion Illiescu, la révolution roumaine en accusation“, ebd, 6 .

63 Souléry, Jean-Claude, Leitartikel „Liberté chérie", in La Dépêche du Midi, (wie Anmerkung 25), 2; Odent, Bruno, „La Thuringe malade d'une réunification qui n'a pas eu et citoyens enracinés“, in L'Express, Dossier „Mur de Berlin. L'Europe, 30 ans après“, (wie Anmerkung 15), 54-55 ; De Barochez, Luc, „Europe. Et si la révolution pacifique de 1989 était à la source du regain identitaire d'aujourd'hui...", in Le Point, Dossier „Chute du Mur, Histoire inattendue“" (52-61), Nr. 2463, (wie Anmerkung 15), 53-56. 
gegenüber Europa und seinen Institutionen werden ausführlich kommentiert ${ }^{64}$, desgleichen die Frage, welche Rolle Russland auf dem europäischen Kontinent zukäme ${ }^{65}$. Manche Artikel behandeln das Thema der Grenzen und Mauern in Europa und beklagen ein in sich verschlossenes Europa und eine Welt, die Mauern bauen würde statt sie zu zerstören $^{66}$ :

Dreißig Jahre ist es nun schon her und es kommt mir so weit weg vor. Zur damaligen Zeit schien die Menschheit Jahr um Jahr auf dem Weg zum Besseren. [...] Dreißig Jahre später ist der Mauerbaumarkt wieder voll am boomen [...]. 1989 spielten wir den schönen Part: Wir waren im Lager der Freiheit und der über den Stacheldraht hinweg gereichten Hände. Sollten wir 1989 dabei sein, auf die falsche Seite der Mauer zu gleiten? Es wäre eine seltsame Wahl, ein Zeichen, dass wir die Lektion von 1989 nicht behalten hätten: Mauern fallen am Ende immer. ${ }^{67}$

Die Kritik an einem Europa, das Mauern errichte, statt sich zur Ehre zu machen, ein Raum der Freiheit und der Demokratie zu sein, findet sich auch in den zahlreichen Artikeln wieder, deren Gegenstand die Bedeutung des Berliner Mauerfalls für die Weltgeschichte und die ideologischen Entwicklungen ist. Ausgangspunkt ist das Aufgeben der ursprünglichen These von Francis Fukuyama über das Ende der Ideologien und den Sieg des Modells der liberalen Demokratie. Das „Ende der Geschichte“ wird als eine nunmehr überholte These bezeichnet ${ }^{68}$, die Vorahnungen des amerikanischen Politikwissenschaftlers wären „dreißig Jahre später" komplett „widerlegt“" 69 :

Ernüchtert sind sich die Menschen im Westen bewusst geworden, dass die Geschichte alles andere als abgeschlossen ist, dass sie da ist, lebt, dass sie von jedem Mut und Durchblick fordert, weil sie gestern wie heute tragisch sein kann. ${ }^{70}$

64 Ricard, Philippe, „Pour une majorité d'Européens de l'Est, la démocratie est en dange Un sondage montre un désenchantement, trente ans après la chute du Mur", in Le Monde, Nr. 23271, 5.11.2019, 5; Mink, Georges, „Les élites populistes perçoivent l'UE comme un nouveau Moscou“, in Cahier du Monde "30 ans après la chute du Mur", Nr. 23276, (wie Anmerkung 39), 7 .

65 Gratchev, Andreï, „En 1989, personne n'était préparé à ce bouleversement historique “, in Le Monde, Nr. 23273, (wie Anmerkung 17), 28

66 Malagardis, Maria, „De l'Arabie Saoudite aux Etats-Unis, 40000 kilomètres de murs", in Libération, Nr. 11954, (wie Anmerkung 25), 8; Farcis, Sébastien, „Les frontières de l'Inde aussi renforcées que contestées", ebd., 9.

67 „Cela fait déjà trente ans et cela me semble si loin. A cette époque, l'humanité semblait aller vers le mieux d'année en année. [...]. Trente ans après, le marché de la construction des murs est à nouveau en plein boom [...]. En 1989, nous avions le beau rôle : nous étion dans le camp de la liberté et des mains tendues par-dessus les barbelés. En 2019, serionsnous en train de passer du mauvais côté du mur? Ce serait un choix étrange, un signe que nous n'aurions pas retenu la leçon de 1989: les murs finissent toujours par tomber Ponce, Anne, Leitartikel, „1989- 2019. De l'autre côté du Mur“, in La Croix, Nr. 41554, (wie Anmerkung 18), 5

68 De Montety, Etienne, „Editorial. L'histoire est toujours là“, in Le Figaro, (wie Anmerkun 14), 1; Wurtz, Francis, „La 'fin de l'histoire' a 30 ans", in L'Humanité, (wie Anmerkung 14), 9; Galtier, Mathieu / Labrutère, Florence / Macé, Célian / Malagardis, Maria / Maurice, Stéphane / Silvestroni, Justine / Witter, Louis, „En 2019, l'Europe compte ses murs“, in Libération, Nr. 11954, (wie Anmerkung 25), 2-5, hier 3.

69 Gibier, Henri, „Nouveaux Murs“, in Les Echos, (wie Anmerkung 14), 11.

70 „Dégrisés, les Occidentaux ont pris conscience que l'histoire, loin d'être finie, était toujours là, vivante, exigeant de chacun courage et lucidité, parce qu'hier comme aujourd'hui, elle peut être tragique“", De Montety, Etienne, (Anmerkung 67).
L'Express veröffentlicht in der Ausgabe vom 6.-12. November ein Interview mit Francis Fukuyama. Letzterer gibt selbst die Grenzen seiner Argumentation über das Ende der Geschichte zu und, ausgehend von den Krisen der liberalen Demokratie und dem von inm nicht vorhergesehenen Erstarken der populistischen Strömungen, schlägt er eine neue Auslegung der Ereignisse vor. ${ }^{71}$ Auch die Dauerhaftigkeit und die Suprematie der liberalen Demokratie als einzigem Modell werden inzahlreichenArtikeln zum 30. Jubiläum des Mauerfalls in Frage gestellt. ${ }^{72}$ Der Abriss der Berliner Mauer und das Erlöschen vieler damit verbundener Hoffnungen bleiben in der Tat der Anlass, eindeutige Unterschiede zwischen den Ideologien zu formulieren und zu verdeutlichen. Wenn auch die These vom Ende der Geschichte widerrufen wird, so wird doch der Zusammenbruch des Ostblocks meistens als Ende der kommunistischen Ideologie interpretiert, mit der Annahme, dass der Kommunismus seinen eigenen Untergang verursacht habe. ${ }^{73}$

Lucien Sève vertritt in L'Humanité den entgegengesetzten Standpunkt: Was 1989 verschwand, sei kein Kommunismus gewesen, der wahre Kommunismus komme erst noch, und zwar in einer Welt, die sich verändern müsse, um zu überleben. ${ }^{74}$ Libération zufolge hätten der Mauerfall und seine Nachwirkungen die Abwendung von einer bipolaren Welt und die Hinwendung zum Süden und zu den Schwellenländern ermöglicht, eine Perspektive und ein Weg, die man weiterverfolgen müsse. ${ }^{75}$ Schlussendlich nimmt die Mehrheit der Artikel in Libération das Mauerfall-Jubiläum zum Anlass, um die Vorstellung einer offenen und humanistischen Welt ohne Mauern und Grenzen zu vertreten. ${ }^{76}$

Zusammenfassend zeigen die Zeitungsartikel, die die These und Widerlegung vom „Ende der Geschichte“ thematisieren, am deutlichsten, wie sehr die Interpretation des Berliner Mauerfalls auch noch 2019 in der französischen Presse ein polemisches, ideologisches und politisches Anliegen bleibt.

71 Fukuyama, Francis, „Le dédain des élites nourrit le populisme“, (das Gespräch führte Marc Epstein), in L'Express, Dossier „Mur de Berlin“ (wie Anmerkung 15), 46-47.

72 Duclos, Michel, „Aujourd'hui, la démocratie libérale n'apparaît plus comme le modèle légitime“, in Le Monde, Nr. 23273, (wie Anmerkung 17), 29.

73 Guetta, Bernard, „Le communisme a tué le communisme“, in L'Obs, Dossier "Cahier Historique. Mur de Berlin. Le jour où le monde a basculé", (wie Anmerkung 27), 4-7; Souléry, Jean-Claude, Leitartikel „Liberté chérie“ (wie Anmerkung 63; Joffrin, Laurent, „, Trouble nostalgie“, in Libération, Nr. 11954, (wie Anmerkung 25), 2.

74 Sève, Lucien, „Le 'communisme' est mort, vive le communisme !“, (das Gespräch führte Pierre Chaillan), L'Humanité, (wie Anmerkung 14), 8.

75 Badie, Bertrand, ,'Avec la chute du Mur, la décolonisation a pris tout son sens : le Sud est soudain devenu central.'“, (das Gespräch führte Catherine Calvet), in Libération, Dossier "Berlin 30 ans, l'Âge Mur" nº 11953, (wie Anmerkung 26), 20-21.

76 Grosser, Pierre, „Empêcher les gens de partir est devenu quelque chose d'obsolète“, (das Gespräch führte Arnaud Vaulerin), Libération, $\mathrm{n}^{\circ}$ 11954, 9.-11.11.2019, 6-7. 